\title{
Procedimentos para obtenção de extratos e determinação de micronutrientes em amostras de solo
}

\section{Proceedings for extracts obtaining and micronutrients determination in soil samples}

\author{
Fábio Rogério Ortiz ${ }^{1}$; Osmar Rodrigues Brito ${ }^{2 *}$; \\ Clóvis Manuel Borkert ${ }^{3}$; Rafael Mizubuti Brito ${ }^{4}$
}

Resumo

\begin{abstract}
Deficiências ou excessos de micronutrientes no solo pode ser diagnosticada por diversos procedimentos. Dentre estes se destaca a análise química, uma vez que possibilita a avaliação prévia da disponibilidade destes nutrientes para as plantas. Apesar de muitos laboratórios realizarem análises para micronutrientes em amostras de solo, a falta de padronização de procedimentos metodológicos pode comprometer a comparação de resultados. Uniformizar procedimentos analíticos é uma necessidade indiscutível, quando se pretende garantir qualidade e credibilidade aos resultados. Este estudo foi realizado com o objetivo de comparar procedimentos analíticos, visando contribuir com novos conhecimentos para definição e padronização metodológica para análises químicas de micronutrientes em amostras de solo. A decantação por dezesseis horas e a filtração com papel Whatman $\mathrm{n}^{\circ} 42$, resultaram na determinação de teores diferentes para os micronutrientes ferro, cobre, zinco e manganês em amostras de solo, não possibilitando uso alternativo. A escolha de um destes procedimentos deve resultar em uma padronização para todos os laboratórios participantes da rede de controle de qualidade.
\end{abstract}

Palavras-chave: Análise de solo, micronutrientes, química do solo

\begin{abstract}
The deficiencies or micronutrients excesses in the soil can be diagnosed by several procedures. Among these, the chemical analysis of the soil is a prominence instrument, because it makes possible the previous evaluation of micronutrients available to the plants. In spite of many laboratories accomplish micronutrients chemical analyses in the soil, the lack of methodologies standardisation can commit the reliability of the results. Analytical procedures standardisation is an unquestionable need, when it intend to guarantee quality and credibility to the results. This study was carried out with the objective of comparing analytical procedures, seeking to contribute with new knowledge for definition and methodological standardisation of micronutrients chemical analyses of soil samples. The procedures of decantation for 16 hours and the filtration with filter paper Whatman 42, showed differents contents for iron, cooper, zinc and manganese in soil samples, don't having possibility for alternative use. The choice of one this procedures it should result in a standardisation to all the participant laboratories of quality control.
\end{abstract}

Key words: Soil analysis, micronutrients, soil chemistry

Técnico da EMBRAPA Soja, Londrina, PR.

2 Docente do Departamento de Agronomia da Universidade Estadual de Londrina. Londrina, PR. Osmar Rodrigues Brito. Universidade Estadual de Londrina.CEP: 86.051-990, Londrina, PR.Departamento de Agronomia. E-mail: osmar@uel.br.Telefone: (43) 3371-4782 .

3 Pesquisador da EMBRAPA Soja. Londrina PR.

4 Acadêmico do Curso de Graduação em Agronomia da UEL. Londrina, PR

* Autor para correspondência 


\section{Métodos de análises químicas do solo}

Os regentes utilizados nas análises químicas devem extrair do solo quantidades de nutrientes que correlacione com as quantidades absorvidas pelas plantas. Entretanto, nem sempre que se propõe uma metodologia analítica atende-se a esta expectativa. Segundo Ferreira \& Cruz (1997), os estudos de métodos compreendem três fases: a primeira, diz respeito à definição ou padronização de parâmetros analíticos; a segunda, tem em vista a avaliação do seu desempenho, como medida de disponibilidade; a terceira, visa à determinação de classes de teores ou limites críticos para interpretação de resultados.

A análise química do solo para macronutrientes, utilizada freqüentemente no Brasil, compreende procedimentos que regionalmente, estão padronizados. Entretanto, ainda não existe padronização para análise de micronutrientes, o que dificulta a comparação e interpretação dos resultados obtidos. As metodologias utilizadas para avaliar a disponibilidade dos micronutrientes, em geral, têm apresentado resultados discordantes e às vezes contraditórios. Este fato pode ser atribuído às diferenças existentes entre as soluções extratoras e as marchas analíticas empregadas, bem como à sensibilidade do método às variações físico-químicas do solo.

Outro fator que pode interferir nos resultados analíticos e que tem implicações prática e econômica refere-se ao uso da decantação em lugar da filtração, pois quando se consegue a substituição, reduz-se o tempo de execução e o custo final das análises.

A decantação foi utilizada, sem prejuízo da qualidade dos resultados por Ferreira e Cruz (1997), quando estudaram diferentes procedimentos para extração de zinco em amostras de solo, utilizando como extrator a solução Mehlich-1.

Segundo Ribeiro e Saraiba (1984), Bataglia e Raij (1989) e Ferreira e Cruz (1997), apesar de muitos laboratórios já realizarem análises de micronutrientes em amostras de solo, a falta de padronização das metodologias de avaliação pode comprometer a confiabilidade dos resultados. Assim, uniformizar procedimentos metodológicos é uma necessidade indiscutível, para se garantir qualidade e atribuir credibilidade aos resultados analíticos das análises químicas de micronutrientes do solo.

Recentemente no Paraná, a Comissão Estadual de Laboratórios de Análises Agronômicas (CELA) assumiu a coordenação do programa de controle de análises de solo do estado. Através desse programa, estão sendo avaliadas e comparadas diferentes metodologias para extração e determinação de micronutrientes em amostras de solo. Alem disso a CELA estabeleceu metas para avaliar a qualidade das análises e o desempenho dos laboratórios.

Na maioria dos laboratórios do Estado do Paraná, utiliza-se solução Mehlich-1, proposta por Nelson e Mehlich (1953), para extrair os micronutrientes Fe, $\mathrm{Cu}, \mathrm{Zn}$ e Mn em amostras de solos. A sua adoção, não implica em custos adicionais e dispensa adaptações da estrutura física dos laboratórios, pois a mesma, já é utilizada rotineiramente para extração de $\mathrm{P}$ e de $\mathrm{K}$ do solo. Dessa forma, este trabalho foi conduzido com o objetivo de comparar procedimentos analíticos, visando, com emprego da solução Mehlich1, a definição e padronização metodológica para análises químicas de micronutrientes em amostras de solo.

\section{Procedimentos experimentais}

Para condução do experimento foram utilizadas amostras de solo coletadas na camada de $0,0-0,2 \mathrm{~m}$, oriundas de áreas experimentais de calibração de micronutrientes, localizadas nas cidades de Mamborê e Ponta Grossa, no Estado do Paraná. Após secagem ao ar, as amostras foram moídas e peneiradas $(2,0 \mathrm{~mm}) \mathrm{e}$, posteriormente, submetidas às análises química para avaliação dos micronutrientes ( $\mathrm{Zn}, \mathrm{Fe}, \mathrm{Cu}$ e $\mathrm{Mn}$ ), utilizando como extrator a solução Mehlich-1 ( $\mathrm{HCl}$ 0,05 $\left.\mathrm{mol} \mathrm{L}-1+\mathrm{H}_{2} \mathrm{SO}_{4} 0,125 \mathrm{~mol} \mathrm{~L}^{-1}\right)$.

$\mathrm{Na}$ extração dos micronutrientes metálicos procedeu-se da seguinte forma: transferiu-se $5 \mathrm{~cm}^{3}$ 
de solo para um erlenmeyer de $125 \mathrm{~mL}$, adicionouse $50 \mathrm{~mL}$ da solução extratora, em seguida, agitouse por cinco minutos em agitador orbital a $250 \mathrm{rpm}$. A partir da extração foram testados dois procedimentos para obtenção do extrato límpido: a) repouso por 16 horas; b) filtração imediata com papel filtro Whatman $n^{\circ} 42$.

Nos extratos obtidos, quantificou-se, por espectrofotometria de absorção atômica os teores de cobre, ferro, zinco e manganês. O delineamento empregado foi o inteiramente casualizado com os tratamentos arranjados em um fatorial 2 x 2 em que os fatores foram dois solos e dois procedimentos para obtenção do extratos límpidos. Para cada tratamento, foram analisadas 20 amostras de solo. Os resultados obtidos foram submetidos a análises de variância e de correlação. Quando necessário, as médias foram comparada pelo teste de Tukey.

Os teores de zinco, ferro, cobre e manganês, obtidos nas amostras dos dois solos utilizados no experimento, encontram-se na Tabela 1 .

Tabela 1. Teores de zinco, ferro, cobre e manganês de dois solos do Estado do Paraná, em função dos procedimentos de obtenção do extrato. ${ }^{1}$

\begin{tabular}{|c|c|c|}
\hline \multirow[t]{2}{*}{ Solos $^{2}$} & \multicolumn{2}{|c|}{ Metodologias } \\
\hline & Filtração & Decantação \\
\hline & \multicolumn{2}{|c|}{ - } \\
\hline Mamborê & $0,70 \mathrm{Ba}$ & $1,27 \mathrm{Ab}$ \\
\hline Ponta Grossa & $0,82 \mathrm{Ba}$ & $1,49 \mathrm{Ab}$ \\
\hline $\mathrm{DMS}=0,12 * *$ & $\mathrm{CV}=13,9 \%$ & \\
\hline & \multicolumn{2}{|c|}{ - Fe, $\mathrm{mg} \mathrm{kg}^{-1}$} \\
\hline Mamborê & $31,99 \mathrm{Bb}$ & $113,35 \mathrm{Aa}$ \\
\hline Ponta Grossa & $47,84 \mathrm{Ba}$ & $90,03 \mathrm{Ab}$ \\
\hline $\mathrm{DMS}=4,87 * *$ & $\mathrm{CV}=10,9 \%$ & \\
\hline & \multicolumn{2}{|c|}{ - } \\
\hline Mamborê & $0,85 \mathrm{Bb}$ & $1,32 \mathrm{Ab}$ \\
\hline Ponta Grossa & $1,41 \mathrm{Ba}$ & $1,59 \mathrm{Aa}$ \\
\hline $\mathrm{DMS}=0,13 * *$ & $\mathrm{CV}=15,8 \%$ & \\
\hline & \multicolumn{2}{|c|}{ - } \\
\hline Mamborê & $9,29 \mathrm{Bb}$ & $27,19 \mathrm{Aa}$ \\
\hline Ponta Grossa & $12,64 \mathrm{Ba}$ & $27,51 \mathrm{Aa}$ \\
\hline $\mathrm{DMS}=1,74 * *$ & $\mathrm{CV}=14,5 \%$ & \\
\hline
\end{tabular}

${ }^{1}$ Médias seguidas da mesma letra, maiúscula nas linhas e minúscula nas colunas, não diferem entre si, pelo teste de Tukey a $1 \%$, como indicado para DMS. ${ }^{2}$ Manborê $=\mathrm{LVd}$, textura média, Ponta Grossa $=$ LVd, textura argilosa.
Para todos os micronutrientes analisados, foram observadas diferenças significativas, entre os procedimentos testados. A decantação por dezesseis horas resultou sempre na determinação dos maiores teores de micronutrientes nos solos estudados (Tabela 1), provavelmente devido ao maior tempo de contato entre o solo e a solução extratora. Estes resultados estão de acordo com o que foi observado por Pereira et al. (2001), que compararam métodos de extração de micronutrientes, em amostras de solos do Estado do Rio de Janeiro.

Nos testes de correlação entre os dois procedimentos, considerando-se as quantidades de micronutrientes das amostras de solos, só foi observado coeficiente de correlação significativo $(\mathrm{r}=0,55)$, para o cobre. Apesar de significativo, esse coeficiente pode ser considerado baixo, o que indica a baixa afinidade entre os procedimentos comparados.

Levando em consideração os resultados apresentados pode-se afirmar que os procedimentos testados são eficientes para avaliação dos micronutrientes testados, porém diferentes, não possibilitando uso alternativo. Sendo assim, a escolha entre um ou outro, deve ser feita levando-se em consideração a praticidade e o custo de execução da análise. Uma vez definido e adotado pela rede de laboratórios, o procedimento deve ser padronizado, para possibilitar a comparação de resultados.

Como a solução Mehlich-1, historicamente vem sendo utilizada para a determinação de $\mathrm{P}$ e de $\mathrm{K}$, quando então se adota a decantação, este deve ser o procedimento a ser escolhido e adotado, para avaliação dos micronutrientes $\mathrm{Fe}, \mathrm{Cu}, \mathrm{Zn}$ e $\mathrm{Mn}$, uma vez que não acarretará alterações significativas na rotina dos laboratórios.

\section{Considerações finais}

A filtração com papel de filtro Whatman $n^{\circ} .42 \mathrm{e}$ a decantação por 16 horas, utilizadas para obtenção de extratos de solo, resultaram em diferentes teores 
de zinco, ferro, cobre e manganês em amostras de solo.

A comparação de resultados analíticos para teores ferro, cobre, zinco e manganês em amostras de solos, só será possível e adequada, quando os laboratórios adotarem os mesmos procedimentos par obtenção de extrato.

A adoção da decantação por 16 horas facilita as análises dos micronutrientes estudados, uma vez que estes poderiam ser avaliados no mesmo extrato utilizado para avaliação do $\mathrm{P}$ e o do K, em amostras de solo.

\section{Referência}

BATAGLIA, O. C.; VAN RAIJ, B. Eficiência de extratores de micronutrientes na análise de solos. Revista Brasileira de Ciência do Solo. Campinas, v. 13, p. 205-210, 1989.
FERREIRA, M. E.; CRUZ, M. C. P. Seleção de extratores químicos para avaliação da disponibilidade de zinco em solos do Estado de São Paulo. Pesquisa Agropecuária Brasileira. Brasília, v. 27, p. 293-304,1997.

NELSON, W. L.; MEHLICH, A.; WINTERS, E. The development, evaluation and use of soil tests phosphorus availability. In: PIERRE, W. H.; NORMAN, A. G. (ed.). Soil and fertilizer phosphorus in crop nutrition. New York: Academic Press, 1953.p. 89-122. (Agronomy. A Series of Monographs, 4).

PEREIRA, M. G.; PÉREZ, D. V.; VALLADARES, G. S.; SOUZA, J. M. P. F.; ANJOS, L. H. C. Comparação de métodos de extração de cobre, zinco, ferro e manganês em solos do estado do Rio de Janeiro. Revista Brasileira de Ciência do Solo. Viçosa, v. 25, p. 655-660, 2001.

RIBEIRO, A. C.; SARABIA, W. A. T. Avaliação de extratores para zinco e boro disponíveis em latossolos do triângulo mineiro. Revista Brasileira de Ciência do Solo. Campinas, v. 8, p. 85-89, 1984. 